\title{
Adenocarcinoma Arising from a Gastric Duplication Cyst
}

\author{
Brian P.H. Chan, BMSc, MD', Martin Hyrcza, MD, PhD², Jennifer Ramsay, MDCM², and \\ Frances Tse, MD, MPH' \\ 'Division of Gastroenterology, McMaster University, Hamilton, Ontario, Canada \\ ${ }^{2}$ Department of Pathology and Molecular Medicine, McMaster University, Hamilton, Ontario, Canada
}

\begin{abstract}
Gastric duplication cysts are rare congenital anomalies, and malignant transformation has only been reported in 11 cases. A healthy 57-year-old woman presented with abdominal discomfort, and computed tomography revealed a $5.8 \times 6.6 \times 8.2 \mathrm{~cm}$ mass at the gastric fundus. On endoscopic ultrasound, the mass was mostly hypoechoic with anechoic cystic cavities arising from the submucosal layer. Fine-needle aspiration was suspicious for adenocarcinoma. Surgical pathology confirmed high-grade adenocarcinoma, clear cell cytology arising from a foregut duplication cyst. Endoscopic ultrasound is underutilized in the evaluation duplication cysts and should be considered in routine workup.
\end{abstract}

\section{INTRODUCTION}

Duplication cysts are rare congenital anomalies, predominantly seen in children, and can occur anywhere along the alimentary tract. The ileum is the most common site, while the stomach and colon are least common.' Gastric duplication cysts (GDC) represent 2-8\% of all cases of duplication cysts.' Most GDCs are asymptomatic, but some may cause mass-related symptoms such as abdominal pain, nausea, or vomiting. Malignant transformation is rare, with only 11 cases described in the literature, with all diagnoses made by surgical resection. We present a case of adenocarcinoma arising from a GDC, with the initial diagnosis made by endoscopic ultrasound (EUS).

\section{CASE REPORT}

A 57-year-old woman presented with a 2-month history of non-specific abdominal discomfort. Past medical history and physical examination were unremarkable. Laboratory tests were all within normal range. Abdominal computed tomography $(C T)$ revealed a fungating, intraluminal mass $(5.8 \times 6.6 \times 8.2 \mathrm{~cm})$ arising from the posterior fundal aspect of the stomach (Figure 1). It was heterogeneously enhancing, and there was no evidence of lymphadenopathy or involvement of adjacent organs. Tumor markers, including CEA and CA 19-9, were normal.

A series of 3 esophagogastroduodenoscopies confirmed a large submucosal mass in the gastric fundus with normal overlying mucosa. Biopsies were non-diagnostic on all occasions. Because of the potential for malignancy, EUS was performed to facilitate diagnosis. On EUS, the mass was mostly hypoechoic with anechoic cystic cavities arising from the submucosal layer with no invasion of the deeper layers of the stomach (Figure 2). The lesion was quite vascular on Doppler and measured $69 \mathrm{~mm}$ in size. The differential diagnosis included benign lesions such as leiomyoma and schwannoma, as well as malignant lesions such as gastrointestinal (GI) stromal tumor (GIST), adenocarcinoma, lymphoma, and neuroendocrine tumor.

Fine-needle aspiration from EUS showed high-grade dysplastic epithelium with cribriform appearance but no overt stromal invasion, in keeping with adenocarcinoma (Figure 3). Staining was positive for CK7 and CDX2. Despite a positive biopsy suggesting adenocarcinoma, GIST was still considered high in the differential because primary

ACG Case Rep J 2018;5:e42. doi:10.14309/crj.2018.42. Published online: June 6, 2018.

Correspondence: Frances Tse, Division of Gastroenterology, Department of Medicine, McMaster University, Room 2F53, 1200 Main St West, Hamilton, ON, Canada L8N 3Z5 (tsef@mcmaster.ca).

(C) $(1) @$ Copyright: ๑ 2018 Chan et al. This work is licensed under a Creative Commons Attribution-NonCommercial-NoDerivatives 4.0 International BY NC ND License. To view a copy of this license, visit http://creativecommons.org/licenses/by-nc-nd/4.O. 


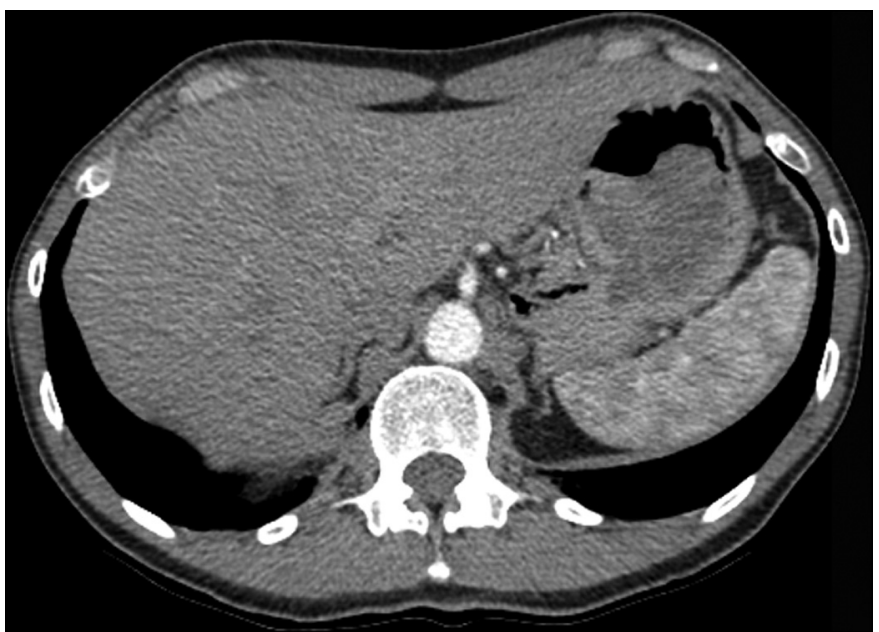

Figure 1. Axial computed tomography showing a large mass projecting intraluminally at the posterior fundal aspect of the stomach.

submucosal tumor-like adenocarcinomas are rare in the $\mathrm{Gl}$ tract. Positron-emission tomography and CT of the chest, abdomen, and pelvis affirmed localized disease. Gross appearance of the transected duplication cyst after laparotomy was an endophytic submucosal mass composed of thickened undulating mucosa and an underlying layer of attenuated muscle (Figure 4). Frozen section analysis confirmed adenocarcinoma. The patient did well after total gastrectomy, distal esophagectomy, and D2 lymphadenectomy with Roux-en-Y reconstruction, and she was discharged home 7 days after surgery.

Surgical pathology confirmed moderately differentiated invasive adenocarcinoma, clear cell variant with a maximum dimension of $2 \mathrm{~cm}$, arising from an $8-\mathrm{cm}$ foregut duplication cyst (Figure 5). The GDC was continuous with the mucosal surface, and it occupied the submucosa but did not involve the underlying muscularis propria. Resection margins were clear with 17 lymph nodes that sampled negative for malignancy (pathological stage $\mathrm{pT} \mathrm{rb}$ NoMX). Immunohistochemistry was positive

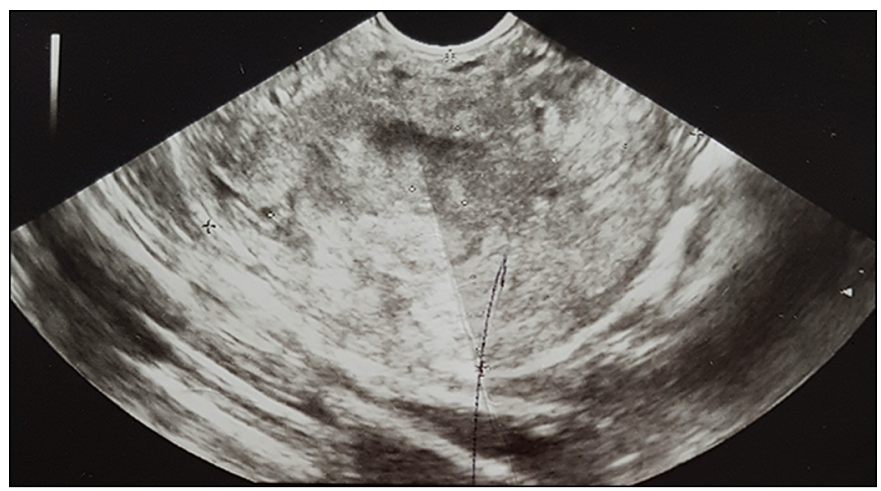

Figure 2. Large, heterogeneous, hypoechoic mass with cystic cavities arising from the submucosal layer of the stomach seen on endoscopic ultrasound (EUS).

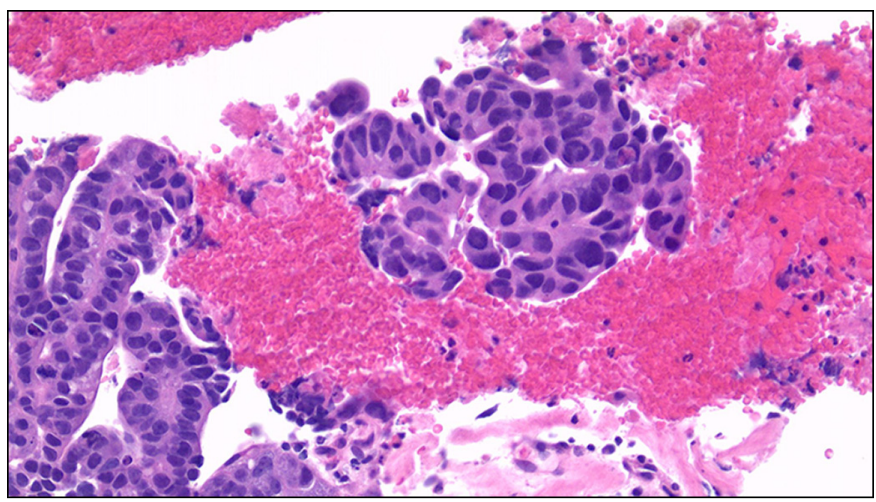

Figure 3. EUS-guided fine-needle aspiration sample showing dehisced atypical epithelium with malignant cytological features consistent with adenocarcinoma (100x).

for p53, villin, and CDX2. Six-month follow-up CT showed no evidence of recurrent or metastatic disease.

\section{DISCUSSION}

Duplication cysts are rare congenital anomalies that can arise anywhere along the alimentary tract. It is typically diagnosed in children before the age of 12 due to symptomatic obstruction. ${ }^{2}$ Pathogenesis remains poorly defined. The most widely accepted theory is abnormal recanalization after solid epithelial stage of embryonic bowel development. ${ }^{2}$ There are 2 morphologic types of duplications, cystic and tubular. Cystic duplications are the most common and have no communication with the bowel lumen. Tubular duplications communicate with the lumen in $75 \%$ of cases.' The ileum is the predominant

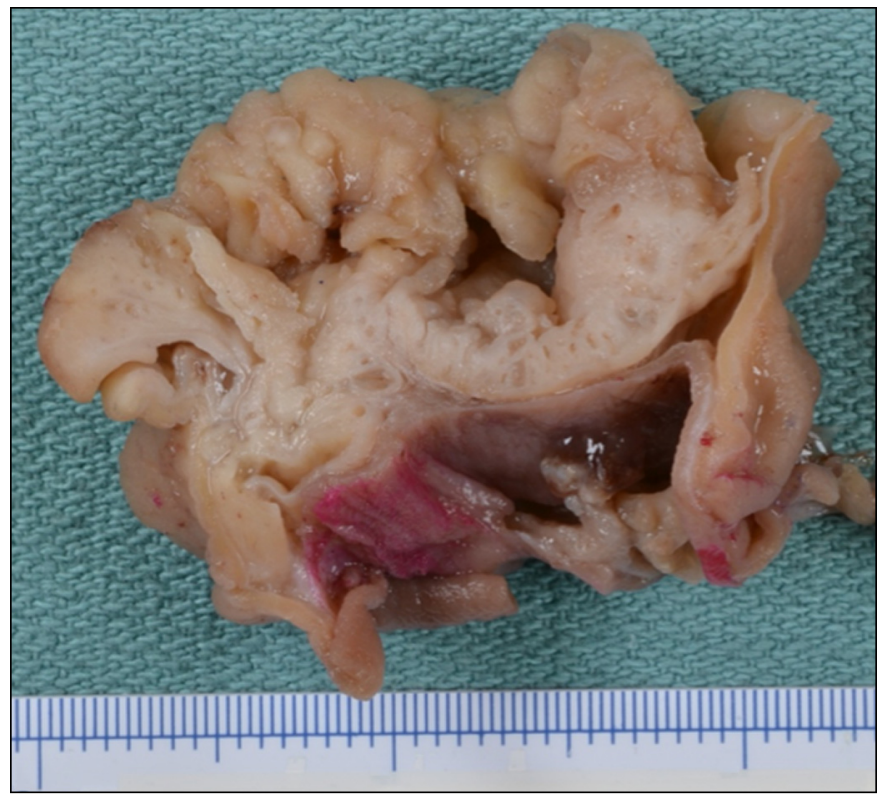

Figure 4. Gross appearance of the transected duplication cyst, an endophytic submucosal mass comprised of thickened undulating mucosa and underlying layer of attenuated muscle. 


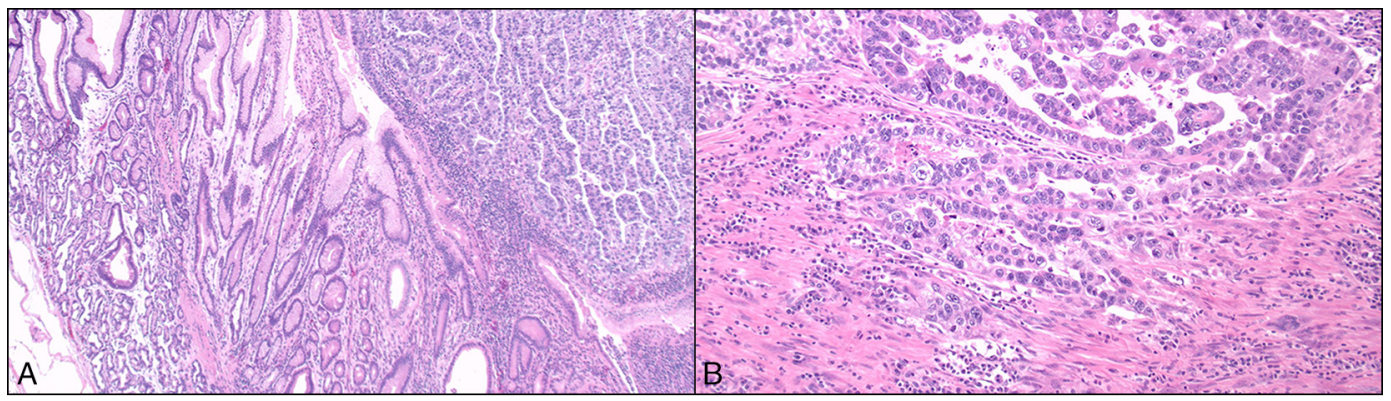

Figure 5. (A) Surgical pathology of thickened mucosa from gastric duplication cyst showing high-grade dysplasia and adenocarcinoma arising in a background of hyperplastic changes with intestinal metaplasia. (B) Further sectioning of the lesion revealing multiple microscopic foci of invasive adenocarcinoma infiltrating submucosa (pathological stage pT1bNo) (100x).

site of duplication, followed by the esophagus, jejunum, stomach, and colon.'

GDCs represent $2-8 \%$ of all cases, and 150 cases have been reported in the medical literature since 1911. ${ }^{2-4}$ The greater curvature is the most common site of GDCs, and they are generally cystic and non-communicating. ${ }^{3}$ Typical features were described in 1959: continuity of the cyst wall within the stomach; the cyst is surrounded by smooth muscle, which is contiguous with the muscle of the stomach; and alimentary tract epithelial lining. ${ }^{3}$ Nearly a third of GDCs are associated with other anomalies such as esophageal and duodenal diverticula, duplication cysts elsewhere, annular and ectopic pancreas, and spinal abnormalities. ${ }^{5}$ Common symptoms of GDCs include abdominal pain, nausea, vomiting, bloating, and constitutional symptoms. Complications range from obstruction, bleeding, fistulization, pancreatitis, to malignant transformation. ${ }^{5}$

Malignant transformation of GDCs is rare, and only 11 cases have been reported in the literature. ${ }^{6-15}$ Adenocarcinoma is the most common histopathology, although squamous cell carcinoma, neuroendocrine, and GIST tumors have been reported. CT is useful for initial diagnosis and can determine cyst size and relationship with other organs. Endoscopy can show a gastric mass, but given the submucosal nature of the cysts, may return non-diagnostic biopsies.

Based on our literature review, EUS appears to be underutilized in the evaluation and diagnosis of GDCs, as all of the previous cases were established with surgical resection. EUS may have an important role in the investigation of duplication cysts because it can differentiate between solid and cystic components, and it can define cyst location in relation to adjacent structures. On EUS, duplication cysts typically appear as anechoic, homogeneous lesions with smooth margins arising from the submucosal layers of the GI tract, although a hypoechoic pattern may also be seen. ${ }^{16}$ EUS-guided fine-needle aspiration can be used to provide a more definitive diagnosis and rule out malignant cyst transformation. ${ }^{17}$ In our rare case of adenocarcinoma arising from a GDC, GIST was ruled out and adenocarcinoma was confirmed. This led to appropriate surgical management without the use of neoadjuvant chemotherapy.

Management of symptomatic lesions is traditionally surgical. Management of asymptomatic lesions remains controversial as the natural history and risk factors associated with malignant transformation remain unclear, although some authors advocate for resection given the potential for malignancy. In this context, EUS may be a useful tool for screening and surveillance of these lesions, and it can help guide management. EUS is underutilized in the diagnosis and management of these lesions, and it should be included in the routine workup of GDCs.

\section{DISCLOSURES}

Author contributions: All authors wrote the manuscript and reviewed the literature. F. Tse is the article guarantor.

Previous presentation: This case was presented in part at United European Gastroenterology Week; October 28November 1, 2017; Barcelona, Spain.

Financial support: None to report.

Informed consent was obtained for this case report.

Received December 12, 2017; Accepted April 4, 2018

\section{REFERENCES}

1. Macpherson RI. Gastrointestinal tract duplications: Clinical, pathologic, etiologic, and radiologic considerations. Radiographics. 1993;13(5):1063-80.

2. Gross RE, Holcomb GW Jr, Farber S. Duplications of the alimentary tract. Pediatrics. 1952;9(4):448-68.

3. Rowling JT. Some observations on gastric cysts. Br J Surg. 1959;46 (199):441-45

4. Cunningham SC, Hansel DE, Fishman EK, Cameron JL. Foregut duplication cyst of the stomach. J Gastrointest Surg. 2006;10(4):620-21.

5. Zheng J, Jing $\mathrm{H}$. Adenocarcinoma arising from a gastric duplication cyst. Surg Oncol. 2012;21(2):e97-101.

6. Mayo HW, McKee EE, Anderson RM. Carcinoma arising in reduplication of the stomach (gastrogenous cyst): A case report. Ann Surg. 1955;141 (4):550-55. 
7. Treiger M, Rubens J, Chindler J, et al. [Stomach duplication. Report of a second case in literature complicated by a peptic ulcer and malignant neoplasms]. Hospital (Rio J). 1969;75(1):1-10.

8. Ishikawa M, Samejima N, Matsushita M, Shibano N, Hishiyama S. A case of early cancer of the duplicated stomach accompanied with gastric cancer. Japanese J Gastroenterol Surg. 1988;21(8):2148-51.

9. Coit DG, Mies C. Adenocarcinoma arising within a gastric duplication cyst. J Surg Oncol. 1992;50(4):274-77.

10. Mamiya N, Karasawa Y, Kojima N, Takemoto T, Kondoh N, Kasai S. [A case of gastric duplication cyst containing papillary adenocarcinoma]. Nihon Shokakibyo Gakkai Zasshi. 1996;93(1):34-38.

11. Kuraoka K, Nakayama H, Kagawa T, Ichikawa T, Yasui W. Adenocarcinoma arising from a gastric duplication cyst with invasion to the stomach: A case report with literature review. J Clin Pathol. 2004;57(4):428-31.

12. Horne G, Ming-Lum C, Kirkpatrick AW, Parker RL. High-grade neuroendocrine carcinoma arising in a gastric duplication cyst: A case report with literature review case report. Int J Surg Pathol. 2007;15(2):187-91.
13. Barussaud M-L, Meurette G, Cassagnau E, Dupasc B, Le Borgne J. Mixed adenocarcinoma and squamous cell carcinoma arising in a gastric duplication cyst. Gastroenterol Clin Biol. 2008;32(2):188-91.

14. Cabrera Fernandez D. Gastrointestinal stromal tumor arising from a gastric duplication cyst. ACG Case Rep J. 2016;3(3):175-77.

15. Yamasaki A, Onishi H, Yamamoto H, et al. Asymptomatic adenocarcinoma arising from a gastric duplication cyst: A case report. Int J Surg Case Rep. 2016;25:16-20.

16. Liu R, Adler DG. Duplication cysts: Diagnosis, management, and the role of endoscopic ultrasound. Endosc Ultrasound. 2014;3(3):152-60.

17. Seijo RS, Lariño NJ, Abdulkader NI, et al. [Adult gastric duplication cyst: Diagnosis by endoscopic ultrasound-guided fine-needle aspiration (EUS-FNA)]. Rev Esp Enferm Dig. 2008;100(9):586-90. 